\title{
REVISÃO BIBLIOGRÁFICA DO USO INDISCRIMINADO DOS ANTI- INFLAMATÓRIOS NÃO ESTEROIDAS (AINES)
}

\author{
BIBLIOGRAPHIC REVIEW OF THE INDISCRIMINATED USE OF NON-STEROID \\ ANTI-INFLAMMATORIES (AINES)
}

\author{
Isabelle Novaes Câmera Santos ${ }^{1}$ \\ Otoniel Sampaio Escobar ${ }^{2}$ \\ Juliana Lima Gomes Rodrigues ${ }^{3}$
}

RESUMO: Um dos fatores que contribui para o uso incorreto dos medicamentos é a falta de conhecimento e orientação adequada. Diante isso, faz-se necessária uma orientação clara sobre os riscos que os medicamentos podem causar, se usados de forma incorreta, no organismo de cada indivíduo. O presente artigo tem como objetivo a orientação sobre o uso correto de medicamentos, da classe dos anti-inflamatórios não esteroidais (AINEs). A maioria desses medicamentos são comercializados sem prescrição, facilitando assim seu acesso para a população, o que favorece a automedicação e o uso incorreto, podendo trazer graves consequências a curto, médio e longo prazo. Foi realizada uma revisão bibliográfica de diversos artigos científicos atuais sobre o assunto a fim de obter dados relevantes para a comunidade acadêmica, como também para os profissionais de farmácia. Desta forma, foi possível constatar que esta classe medicamentosa é utilizada de forma irracional pela população o que leva a causar desconfortos físicos e até problemas de saúde mais sérios como úlcera gástrica e duodenal, anorexia, etc.

Palavras-chave: Anti-inflamatórios não esteroidais; Uso incorreto; Automedicação.

ABSTRACT: One of the factors that contributes to the incorrect use of medicines is the lack of knowledge and adequate guidance. In view of this, clear guidance on the risks that drugs can cause, if used incorrectly, in each individual's body is necessary. This article aims to provide guidance on the correct use of drugs, of the class of non-steroidal antiinflammatory drugs (NSAIDs). Most of these drugs are sold without prescription, thus facilitating their access to the population, which favors self-medication and incorrect use, and can have serious consequences in the short, medium and long term. A bibliographic review of several current scientific articles on the subject was carried out in order to obtain relevant data for the academic community, as well as for pharmacy professionals. Thus, it was possible to verify that this drug class is used irrationally by the population, which leads to physical discomfort and even more serious health problems such as gastric and duodenal ulcers, anorexia, etc.

\footnotetext{
I Discente do curso de Farmácia da Universidade Salvador. E-mail: isabellenovaes98@hotmail.com

${ }^{2}$ Discente do curso de Farmácia da Universidade Salvador. E-mail: otonielsampaioescobar@gmail.com

${ }^{3}$ Docente do curso de Farmácia da Universidade Salvador. E-mail: juliana.rodrigues@unifacs.br.
} 
Keywords: Nonsteroidal anti-inflammatory drugs; Incorrect use; Self-medication 


\section{INTRODUÇÃO}

Os anti-inflamatórios não esteroidais (AINEs) são uma classe de medicamentos de uso terapêutico de bastante interesse, devido as suas propriedades analgésicas, antiinflamatórias e antipiréticas, tanto para os profissionais de saúde quanto para os pacientes.

Essa classe de medicamentos é dividida em inibidores não-seletivos da COX e inibidores seletivos da COX-2. De acordo com Vieira (2017) atualmente são conhecidas as COXs I, 2 e 3, a COX-3 foi recentemente descoberta. Sendo a COX-I uma enzima constitutiva que atua na produção de prostaglandinas, enquanto a $\mathrm{COX}-2$ é não constitutiva e tem sua expressão ampliada, sobretudo, nos processos inflamatórios.

A COX-3 é uma variação da COX-I é uma enzima que tem ação inibitória de drogas antipiréticas analgésicas e potencialmente inibidas pelos AINEs, essa inibição pode ocorrer em um processo primário onde essas drogas podem diminuir a dor e possivelmente a febre (SANDOVAL, 2017).

Esses medicamentos são constantemente prescritos por serem de fácil acesso a população, além de serem adquiridos pelos pacientes sem receitas. O elevado custo da consulta médica e o fácil acesso a medicamentos nas farmácias, contribui para esta prática, além das propagandas que incentivam o uso irracional dos medicamentos (Rodrigues; Silva (2014 apud Rankel; Sato; Santiago, 2016).

Como todo medicamento, os AINEs podem causar reações adversas. Para Rankel; Sato; Santiago (2016) isso ocorre devido a sua toxicidade sobre vários sistemas, destacandose como efeitos mais importantes os danos gastrointestinais, que podem ir do desconforto abdominal até a erosão da mucosa, podendo levar ao sangramento e perfuração.

Esses fármacos se não forem usados de forma correta, podem ocasionar problemas de saúde mais graves, como agravamento da hipertensão arterial, insuficiência renal, ou outras doenças relacionadas ao sistema renal. Segundo Sandoval (2017) os AINEs podem provocar uma série de efeitos colaterais como diarreia, hemorragia gastrointestinal, úlcera péptica, disfunção e falência renal, inibição da agregação plaquetária, etc.

Como forma de proteção para a população e orientação sobre o uso correto dos medicamentos, foi criado o Projeto de Lei no 5443/2019, que pretende alterar a Lei 9.782/99, originando assim a modalidade de medicamentos tarja azul permitindo que os farmacêuticos possam prescrevê-los. A tarja azul deverá incluir aqueles medicamentos de tarja vermelha sem retenção de receita que possuem, no mínimo, 70\% dos critérios estabelecidos para os medicamentos isentos de prescrição (CONSELHO FEDERAL DE FARMÁCIA, 2020).

Através desse projeto o farmacêutico terá a autonomia para fazer um melhor acompanhamento e ter um contato mais próximo ao paciente. Segundo Bismark (2019) o farmacêutico sempre, durante toda a sua existência, indica ou prescreve de algum modo produtos medicamentosos para tratamento de doenças, dores ou sintomas até um certo grau de gravidade.

Assim, o presente trabalho abordou como tema o uso indiscriminado dos antiinflamatórios não esteroidais (AINEs). Neste sentido, o problema que orientou o seu desenvolvimento foi: quais os riscos do uso indiscriminado dos anti-inflamatórios não esteroidais? Para tanto, foi definido como objetivo geral realizar revisão de literatura sobre as principais consequências do uso indiscriminado dos anti-inflamatórios não esteroidais (AINES). 
Os anti-inflamatórios não esteroidais podem ser entendidos como uma das classes de medicamentos mais empregadas pela população. São muito utilizados no tratamento da dor, inflamação, febre. Conforme Lima; Alvim (2018) eles possuem ação anti-inflamatória, analgésica e antipirética por inibição da síntese de prostaglandinas mediante ao bloqueio da dessa classe de medicamentos e o cuidado que se deve ter na hora de realizar a dispensação.

\section{METODOLOGIA}

O presente trabalho se trata de uma revisão bibliográfica sistemática, na qual foram utilizadas publicações eletrônicas de periódicos encontrados no Google Acadêmico, escritos nos idiomas português, inglês ou espanhol, publicadas durante o período de 2011 a 202I. Foram empregadas as palavras-chaves: anti-inflamatórios não esteroidais, uso incorreto, automedicação.

Como critérios de exclusão, pode-se mencionar as publicações que não tinham relação com os anti-inflamatórios não esteroidais, as reações adversas causadas por eles, como também os medicamentos isentos de prescrição, outrossim, trabalhos que não foram escritos nos períodos mencionados e que não estavam nos idiomas escolhidos.

\section{RESULTADOS E DISCUSSÃO}

A partir do tema abordado e tendo como base a metodologia, foi empregado como método de busca a palavra-chave "anti-inflamatórios não esteroidais" em conjunto com "uso incorreto" ou "automedicação". A escolha dos artigos foi feita através do Google Acadêmico. Ao todo, foram escolhidos 17 trabalhos relativos com os objetivos específicos e obedecendo aos critérios de exclusão e inclusão. Conforme figura I

Figura I - Fluxograma dos artigos escolhidos

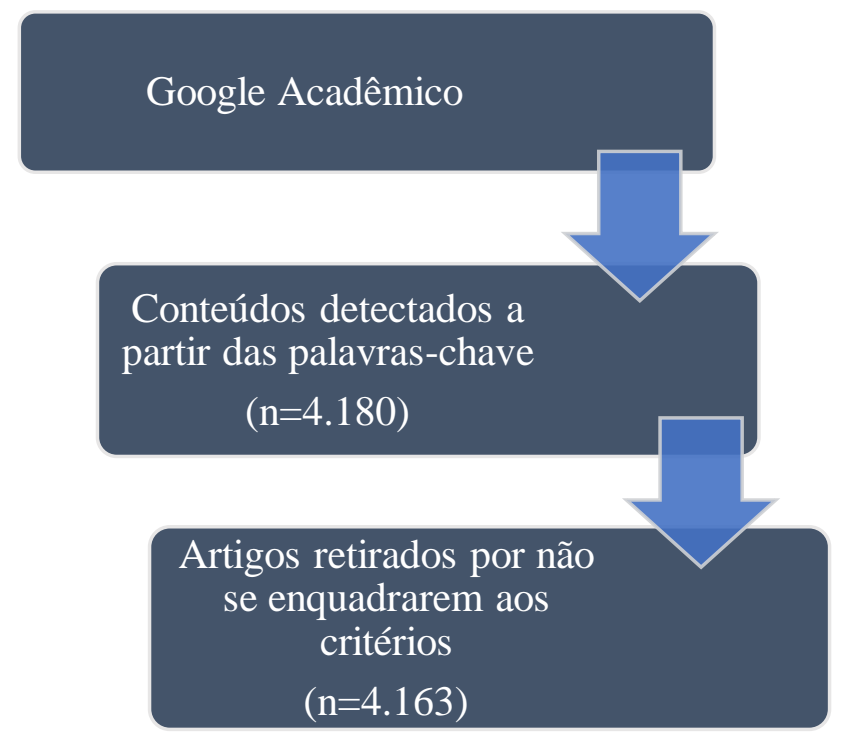

Fonte: Autoria própria 
Os anti-inflamatórios não esteroidais podem ser entendidos como uma das classes de medicamentos mais empregadas pela população. São muito utilizados no tratamento da dor, inflamação, febre. Conforme Lima; Alvim (2018) eles possuem ação anti-inflamatória, analgésica e antipirética por inibição da síntese de prostaglandinas mediante ao bloqueio da ciclo-oxigenase I (COX-I) e ciclo-oxigenase $2(\mathrm{COX}-2)$. Nos dias atuais são conhecidas as COXsi, 2 e 3 (descoberta recentemente).

A COX I é constitutiva, ela é produzida em células dos rins, útero, estômago, sistema nervoso central. Essa isoforma está em constante produção por essas células, elas não necessitam de uma indução. Já a COX 2 pode ser constitutiva, assim como a COX-I está em constante produção, que induzida, a qual é encontrada em macrófagos, linfócitos, polimorfonucleares (PMN), endotélio vascular, ela tem esse nome (COX-2), pois quem induz são as citocinas e outros processos inflamatório.

De acordo com Ko; Albuquerque (2018) a COX-I está associada à produção de prostaglandinas, responsável pela resposta de diferentes efeitos fisiológicos e a COX-2 está expressa principalmente por células envolvidas no processo inflamatório e diante desse quadro inflamatório, sua expressão é aumentada cerca de vinte vezes ou mais. As células que produzem a COX-ı e a COX-2 podem ser observadas na figura 2.

Figura 2 - Células que produzem a COX-1 e a COX-2
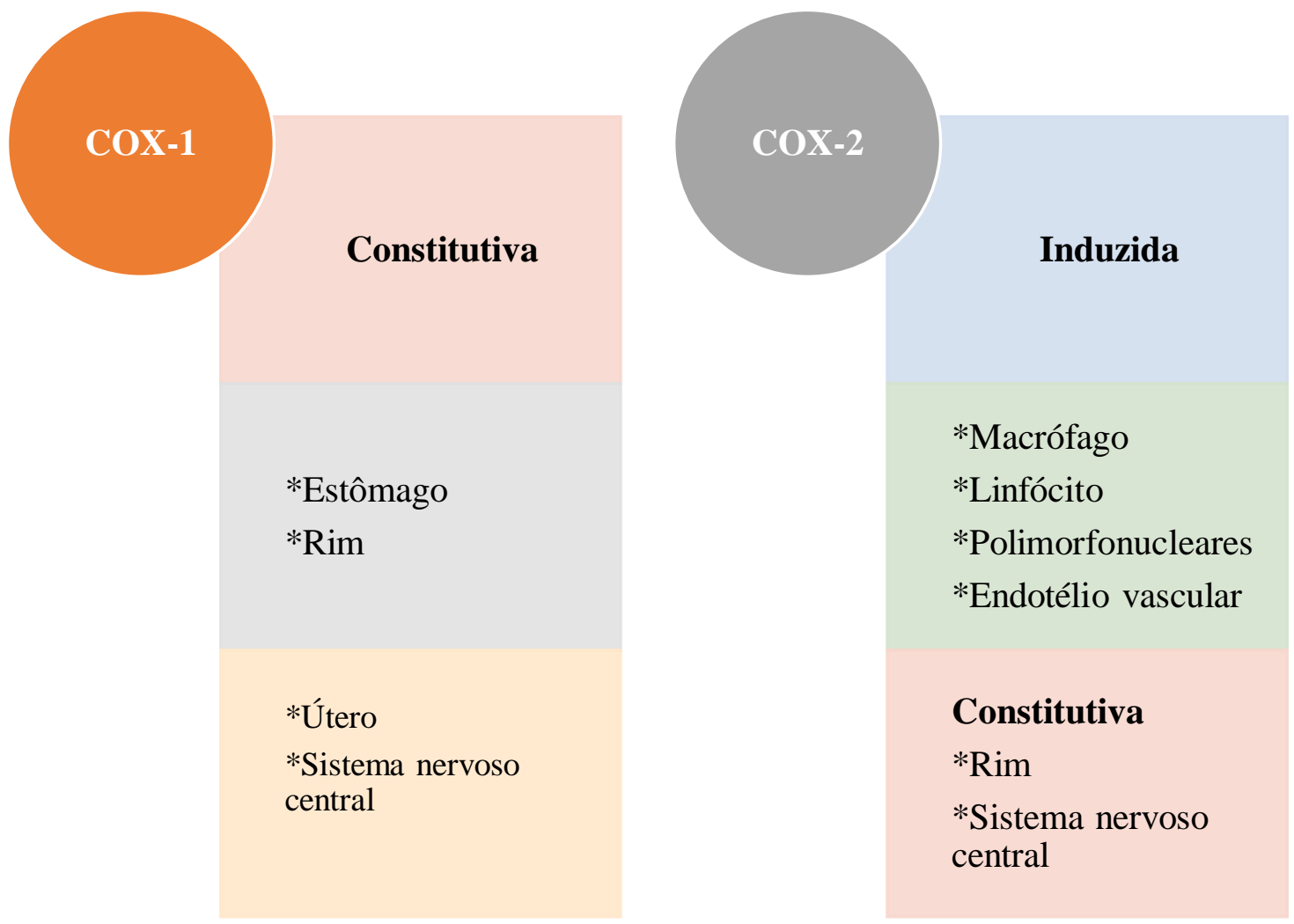

Fonte: Autoria própria 
Há vários tipos de anti-inflamatórios não esteroidais com variados graus de inibição de COX-ı e COX-2 e seus efeitos colaterais são definidos pelo grau de inibição de cada isoenzima (SYLVESTER, 2019). Há fármacos inibidores que são seletivos e fármacos não seletivos das COXs I - 2. Os inibidores da COX-2 são fármacos que geralmente, apresentam menos efeitos adversos, principalmente em relação ao trato gastrointestinal.

Os AINEs se classificam de acordo com Sandoval (2017) em inibidores potentes da COX-I (ácido acetilsalicílico, ibuprofeno e indometacina); inibidores seletivos da COX-2 (celecoxibe, rofecoxibe), porém possuem atividade de inibição sobre a COX-I; e inibidores específicos da COX-2, não agem sobre a COX-I. Tal classificação pode ser observada no quadro I.

Quadro I - Classificação dos AINEs

\begin{tabular}{|c|c|c|c|}
\hline \multicolumn{5}{|c|}{ Classificação dos AINEs } \\
\hline $\begin{array}{c}\text { Inibidores } \\
\text { Seletivos COX-1 }\end{array}$ & $\begin{array}{c}\text { Inibidores Não } \\
\text { Seletivos } \\
\text { COX-1 }\end{array}$ & $\begin{array}{c}\text { Inibidores Não } \\
\text { Seletivos } \\
\text { COX-1 COX-2 }\end{array}$ & $\begin{array}{c}\text { Inibidores Altamente } \\
\text { Seletivos } \\
\text { COX-2 }\end{array}$ \\
\hline ASS $(<100 \mathrm{mg})$ & Indometacina & Nimesulida & Valdecoxibe (Bextra) \\
\hline & Piroxicam & Etodolaco & Celecoxibe (Vioxx) (Celebra) \\
\hline & Diclofenaco & & Lumiracoxibe (Prexige) \\
\hline & Ibuprofeno & & Etoricoxibe (Arcoxia) \\
\hline & & & Parecoxibe \\
\hline
\end{tabular}

Fonte: Adaptado de Batlouni (2010 apud Sandoval, 2017)

O uso indiscriminado de medicamentos pode produzir efeitos não esperados. Como qualquer outro medicamento, os AINEs podem ocasionar reações adversas. Segundo Rang; Dale (20II apud Rankel; Sato; Santiago, 2016) a reação adversa mais comum dos AINEs é a gastrintestinal, resultado principalmente da inibição da COX-I, enzima responsável pela síntese das Prostaglandinas (PGs) que inibem a secreção de ácido gástrico e protegem a mucosa. Esses efeitos estão descritos na figura 3. 
Figura 3: Inibição da COX-I

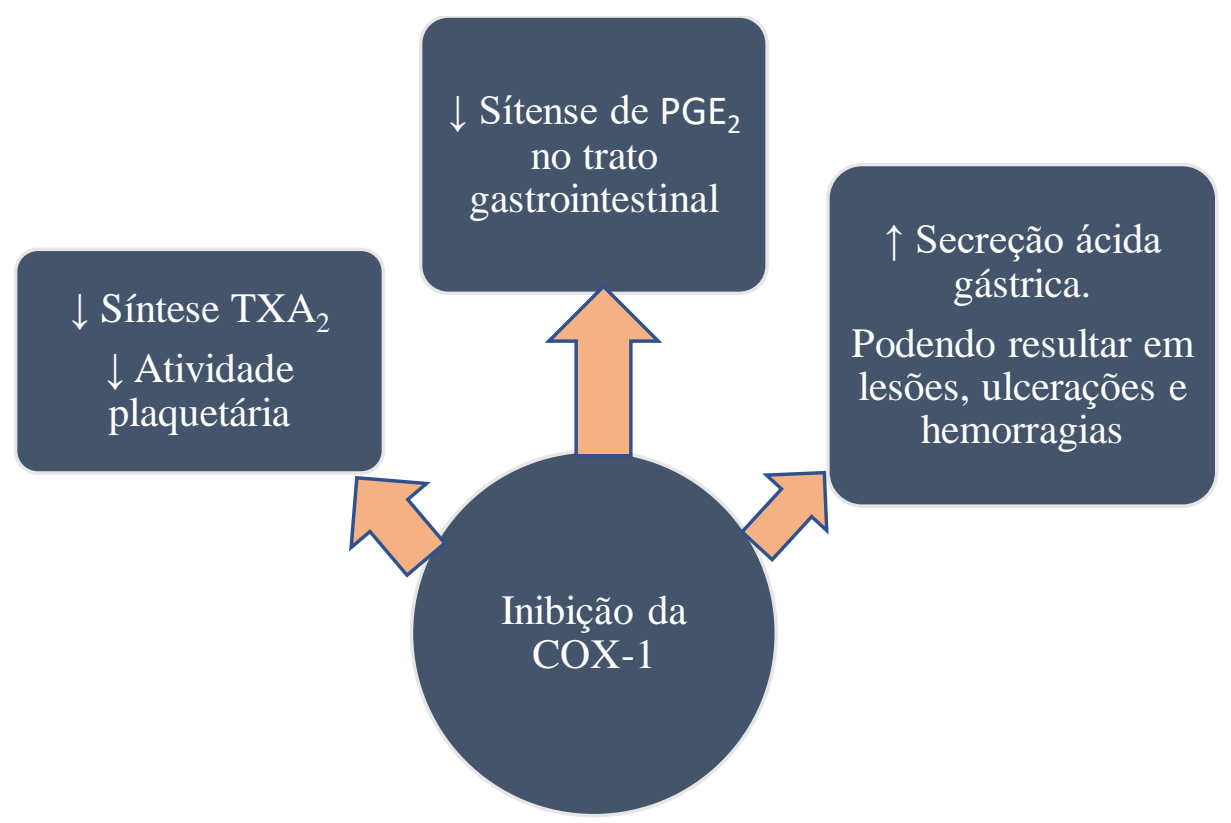

Fonte: Autoria própria

As reações gastrointestinais mais comuns pelo uso incorreto dos AINEs são erosões, ulceração gástrica, náuseas, etc. Segundo com Ko; Albuquerque (2018) os efeitos mais comuns dos AINEs são a nível gastrointestinal. Os efeitos incluem eritema e erosões, úlcera gástrica e duodenal, dispepsia, dor epigástrica, náuseas e vômitos, anorexia, flatulência, e diarreia com possível perda de sangue pelo tubo digestivo.

$\mathrm{Na}$ pesquisa feita por Rankel; Sato; Santiago (2016) em um total de Ioo participantes sobre as reações adversas que tiveram devido ao uso dos AINEs, 59\% relataram ter dores de estômago; $14 \%$ dores de cabeça; $14 \%$ náuseas/vômitos; $9 \%$ dois ou mais sintomas dos já citados e $4 \%$ outros ou não sentiram reações.

Em outro estudo de Pargana (2015) com 27 pessoas ainda sobre reações adversas, elas informaram que das queixas gástricas, destaca-se a azia com $30 \%$ dos inquiridos, seguido de dor abdominal epigástrica e dispepsia ambos referidos em $15 \%$ dos casos, depois a flatulência com uma percentagem de $12 \%$. As náuseas e melena são indicados em $8 \%$ dos casos, os vômitos e outros sintomas representam, cada um, $5 \%$ do universo de todas as queixas e a dor/cólica abdominal apenas $2 \%$.

Observa-se que os AINEs também podem causar diversas reações, e assim seu uso deve ser de forma racional. Com a finalidade de evitar reações adversas, sobretudo as gastrointestinais, foram desenvolvidos fármacos com melhor seletividade de inibição da COX-2. Conforme Oliveira (2019) os inibidores altamente seletivos da COX-2 foram inicialmente uma grande promessa no mercado devido à diminuição dos efeitos gastrointestinais. Os medicamentos são importantes, pois podem salvar vidas. Eles precisam ser utilizados de forma correta e com a finalidade específica para tratar um determinado sintoma. 
De acordo com estudo de Fiuza (2019) Ioo pessoas responderam sobre os motivos que levaram a utilizar os AINEs, aproximadamente $21 \%$ dos entrevistados fizeram o uso desses medicamentos devido às dores em geral, $13 \%$ para inflamação, $12 \%$ para febre, 10\% para garganta, $8 \%$ dor de dente, $7 \%$ dor de cabeça e $7 \%$ coluna.

Corroborando com o estudo acima, Barros; Silva Júnior; Souza Neto (2012), dos 299 participantes $9,7 \%$ tomaram esses fármacos para tratar a lombalgia, 8,7\% para a cefaleia, $7,4 \%$ para febre e dor no corpo, 5,4\%, para dor no corpo, 4,7\%, para cólica menstrual, 4,7\% para dor de dente, 4,3\% para inflamação da garganta. Segue a tabela I abaixo.

Tabela I- Patologia que levaram os pacientes a usar algum tipo de AINEs

\begin{tabular}{|lr|}
\hline Patologia & Porcentagem \\
\hline Lombalgia & $9,7 \%$ \\
\hline Cefaleia & $8,7 \%$ \\
\hline Febre e dor no corpo & $7,4 \%$ \\
\hline Dor no corpo & $5,4 \%$ \\
\hline Cólica menstrual & $4,7 \%$ \\
\hline Dor de dente & $4,7 \%$ \\
\hline Inflamação da garganta & $4,3 \%$ \\
\hline
\end{tabular}

Fonte: Adaptado de Barros; Silva Júnior; Souza Neto (2012).

A partir desses dados, notou-se que a maioria da população utiliza os AINEs para tratar algum tipo de dor, só que esse uso indiscriminado pode apenas mascarar os sinais ou sintomas mais graves de uma doença. Qualquer medicamento pode proporcionar risco à saúde quando empregado sem orientação correta, inclusive os AINEs. De acordo com Ko; Albuquerque (2018) alguns representantes dos AINES estão classificados como MIPs. É natural que boa parte da busca por esses medicamentos pela população seja de livre espontaneidade, e que a automedicação se faz presente em grande parte dos casos.

Em estudos realizados por Bispo (2017) I80 pessoas foram entrevistadas, dessas I70, ou seja, $94 \%$ afirmaram comprar medicamentos sem prescrição médica (MIP). Esses medicamentos são bastante empregados em enfermidades agudas de baixa gravidade, como resfriado, cefaleia, cólicas, etc. $\mathrm{O}$ indivíduo usa esses medicamentos, geralmente, sem ter conhecimento de sua utilidade e os seus fins terapêuticos, ou seja, fazem a automedicação.

A Organização Mundial da Saúde define automedicação como a seleção e uso de medicamentos por indivíduos para tratar doença ou sintomas reconhecíveis, sendo um elemento do autocuidado (WHO, I998 apud MIRANDA FILHO, 2018).

A automedicação deixa a pessoa exposta a diversos riscos de saúde pelo uso indiscriminado de medicamentos, podendo até ocultar uma enfermidade, aumentar seu quadro clínico ou surgimento de outros problemas relacionados à saúde.

Ao avaliarem 428 pessoas através de um questionário on-line entre diferentes participantes do Brasil, Ko; Albuquerque (2018) chegaram ao resultado de que $77 \%$ dos avaliados realizaram uso regular de AINES através da automedicação. De acordo com o gráfico I abaixo. 
Gráfico I - Questionário sobre automedicação

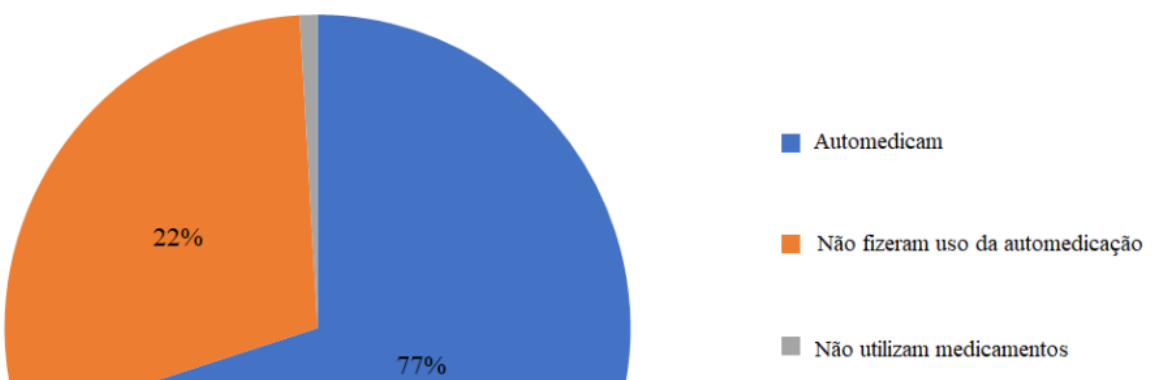

Fonte: Autoria própria

Outro estudo realizado sobre o uso do AINEs sem uma correta orientação profissional demonstrou que das 389 pessoas, 292 pessoas $(75,06 \%)$ disseram ter praticado a automedicação. Sendo que a maior influência foi por familiares $(32,78 \%)$, propagandas veiculadas na mídia $(24,48 \%)$, amigos e vizinhos (22,82\%), indicado por balconistas de farmácias e drogarias (19,91\%). Conforme tabela 2 (ALMEIDA JUNIOR; KAMONSEKI; FERREIRA, 2016).

Tabela 2 - Indicação de medicamentos

\begin{tabular}{cc}
\hline Questões sobre medicamento & $(\%)$ \\
\hline Indicação do medicamento & \\
Familiares & 32,78 \\
Propagandas/mídias & 24,48 \\
Amigos/vizinhos & 22,82 \\
Farmácias/drogarias & 19,91 \\
Reutilizou antigas receitas & 17,47 \\
\hline
\end{tabular}

Fonte: Adaptado de Almeida Junior; Kamonseki; Ferreira (2016)

Diante do exposto, a utilização de medicamentos sem orientação médica ou farmacêutica, como também o seu uso indiscriminado pode gerar um enorme problema de saúde pública. Um dos papéis do farmacêutico é orientar a utilização correta dos medicamentos. Segundo Soterio; Santos (2016) o farmacêutico tem papel fundamental na etapa de orientação da população para o uso correto de medicamentos, dispensação segura, como usar a dose correta, o tempo de tratamento, riscos ou benefícios. 
Proteger o indivíduo da automedicação, informando-o sobre os riscos, oferecer uma maior conscientização sobre o uso correto de medicamentos, fazer uma avaliação do paciente, e se houver necessidade encaminhá-lo a um médico são atitudes importantes do profissional farmacêutico para que haja o emprego consciente e seguro do medicamento.

Em pesquisa realizada sobre orientação farmacêutica Rankel; Sato; Santiago (2016) mostraram que de ioo entrevistados, apenas $16 \%$ relataram procurar o farmacêutico. Para garantir a segurança do paciente e melhorar a qualidade de vida, o farmacêutico deve orientar e informar sobre a correta utilização do medicamento para que haja as menores reações adversas possíveis e uma melhor eficácia terapêutica.

Em outro estudo feito por Silva e Lourenço (2014) observou-se que o profissional farmacêutico foi pouco procurado (apenas $5 \%$ do grupo de roo pessoas). Nota-se que o cidadão precisa entender que o farmacêutico é um profissional da saúde capacitado a auxiliá-lo com seus medicamentos.

Gráfico 2 - Fonte de orientação para a escolha do anti-inflamatório

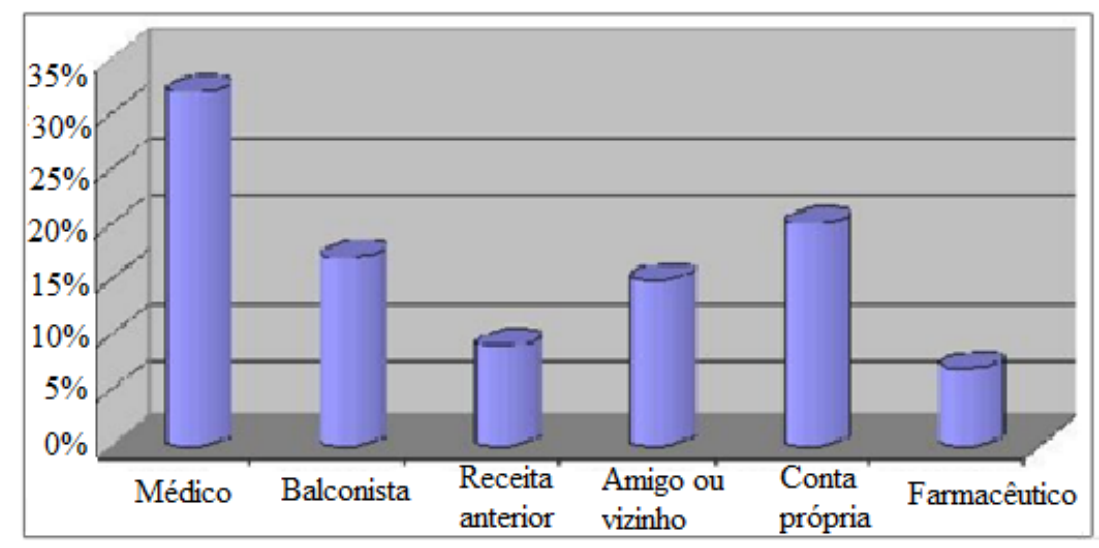

Fonte: Adaptado de Silva; Lourenço (2014)

O acompanhamento farmacoterapêutico é importante para o uso racional de medicamentos, como os AINEs, pois contribui no processo de informação ao usuário como a automedicação, evita custos desnecessários à saúde, efeitos adversos (BANDEIRA, 2013 apud VIEIRA, 2017).

O farmacêutico é muito importante na avaliação do paciente ao decorrer da compra do medicamento, pois ele pode orientar sobre o perigo da automedicação e informar a respeito da utilização de medicamentos de forma correta e responsável. Visando uma proteção maior para o paciente sobre o emprego correto da medicação, foi criado o Projeto de Lei no 5443/2019, que pretende alterar a Lei 9.782/99, gerando uma categoria de medicamento, a tarja azul que os farmacêuticos poderão prescrever.

Esse projeto pretende instituir a modalidade de medicamentos tarja azul (sob prescrição farmacêutica e médica) e possibilitar que farmacêuticos revalidem receitas de antibióticos e medicamentos tarja vermelha sem retenção (CONSELHO FEDERAL DE FARMÁCIA, 2020).

Com a regulamentação da tarja azul e da revalidação da prescrição pelo farmacêutico, o indivíduo pode passar a buscar mais esse profissional de saúde evitando o uso irracional de medicamento ou a automedicação. Segundo Bismark (2019) o que se 
pretende é assegurar que o farmacêutico possa ser reconhecido e identificado pela população como um profissional capaz de cuidar da saúde humana através de medicamentos até certa medida eficiente e segura.

Destaca-se que o farmacêutico não substituirá a prescrição médica inicial para o uso de antibiótico. Ele dará continuidade (se for necessário) por 30 dias, a partir da data que foi dada a receita pelo médico. Sendo assim, a orientação farmacêutica é muito importante, pois trabalha com prevenção e conscientização do paciente, como também colabora para uma melhor saúde e qualidade de vida da população, pois pode conduzir ao efeito terapêutico pretendido e as reações adversas que venham a ocorrer sejam as mínimas possíveis.

\section{CONSIDERAÇÕES FINAIS}

O uso indiscriminado de AINEs é preocupante, visto que a população utiliza esses fármacos sem uma orientação adequada. De acordo com os dados obtidos, somente $5 \%$ das pessoas procuram o farmacêutico para uma orientação correta dos medicamentos. A comercialização desses medicamentos sem a instrução de um profissional adequado pode ser futuramente um grande problema para a saúde pública.

$\mathrm{O}$ uso incorreto dessa classe medicamentosa pode ser um grande fator para a ocorrência de diversos problemas de saúde do indivíduo (como úlcera gástrica e duodenal, anorexia, etc.), principalmente relacionado a reações adversas gastrointestinais. Segundo a pesquisa sobre as reações adversas devido ao uso dos AINEs, 59\% dos entrevistados relataram ter dores de estômago.

Nota-se que os AINEs são muito utilizados na automedicação. Isso fica claro nos estudos onde $77 \%$ dos avaliados realizaram uso regular desses medicamentos através da automedicação. Visando o uso correto desses medicamentos pela população, foi criado o Projeto de Lei 5443/2019, conhecido como Projeto de Lei dos medicamentos de Tarja Azul, o qual, caso seja aprovado, proporcionará ao profissional farmacêutico a orientação e prescrição dos medicamentos dessa e de outras classes medicamentosas,

Cabe ressaltar que para uma melhor eficácia dos medicamentos, faz-se necessário a utilização de forma correta, visto que medicamentos utilizados sem a orientação adequada podem causar diversos danos à saúde, alguns até de forma irreversíveis. $O$ profissional farmacêutico possui um vasto conhecimento sobre medicamentos, o que o qualifica verdadeiramente para tal orientação, assegurando assim a população para que haja a utilização de forma segura e eficiente, na medida certa e com eficácia no tratamento.

\section{REFERÊNCIAS}

ALMEIDA JUNIOR, Geraldo de; KAMONSEKI, Danilo Harudy; FERREIRA, Sandro Rostelato. Perfil de automedicação no município de São Miguel Arcanjo/SP. Revista de Saúde Pública do Paraná, Londrina, v. 17, n. 2, p. 93-1oo, dez/2or6. Disponível em: $\langle$ http://espacoparasaude.fpp.edu.br/index.php/espacosaude/article/view/289/8〉. Acesso em 26 abr. 2021.

BARROS, Nathan Macsuel Oliveira; SILVA JÚNIOR, Nelson Fernandes da; SOUZA NETO, 
Menandes Alves de. Uso de anti-inflamatórios não esteroidais (AINEs) em uma rede privada na população de Carmo do Rio Verde - GO. 2012. 29f. Trabalho de Conclusão de Curso (Graduação) - Faculdade de Ceres, Ceres, 2012. Disponível em: $<$ http://45.4.96.19/bitstream/aee/16762/I/Nathan\%20Barros\%20e\%20Nelson\%20Junior\%20 -\%20Uso\%2ode\%2oAnti-

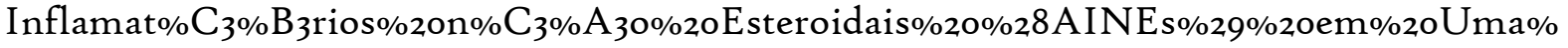

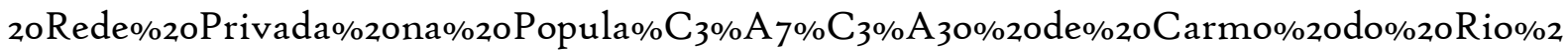
oVerde\%20-\%20GO.pdf $>$. Acesso em 03 maio 2021.

BISMARCK, Eduardo. PROJETO de LEI № 5443 de 2019. Disponível em: <https://www.camara.leg.br/proposicoesWeb/prop_mostrarintegra?codteor $=1818743 \&$ file name $=\mathrm{PL}+5443 / 2019 />$. Acesso em 23 mar. 2021.

BISPO, Naiara Santos et al. Automedicação: solução ou problema? In: XVI SEPA Seminário Estudantil de Produção Acadêmica, 2017, Salvador, Bahia. Disponível em:

〈https://revistas.unifacs.br/index.php/sepa/article/view/4899/3484〉. Acesso em 26 abr. 2021.

CONSELHO FEDERAL DE FARMÁCIA. Brasil poderá ter uma nova categoria de medicamentos. Disponível em: $\left\langle\right.$ https://www.cff.org.br/noticia.php? id $=5640 \&$ titulo $=$ Brasil + poder $\% \mathrm{C}_{3} \% \mathrm{~A}$ I + ter + uma + nov $\mathrm{a}+\mathrm{categoria}+\mathrm{de}+$ medicamentos $>$. Acesso em 24 mar. 2021.

FIUZA, Elizenilda Barbosa Santana. Automedicação com anti-inflamatórios não esteroidais (AINEs) em drogarias de Cruz das Almas-BA. Monografia (Graduação) Faculdade Maria Milza, Governador Mangabeira, 2019. Disponível em:

〈http://famamportal.com.br:8o82/jspui/bitstream/123456789/1921/r/TCC\%2oELIZENIL

DA\%20\%2813-01\%29.pdf >. Acesso em 26 abr. 2021.

KO, Lynkon Tin Yang; ALBUQUERQUE, Cristina Northfleet de. A evolução do mercado de anti-inflamatórios não esteroidais (AINEs) e o papel do farmacêutico frente à automedicação. 2018. 59f. Trabalho de Conclusão do Curso (Graduação) - Universidade de São Paulo, 2018. Disponível em: 〈https://repositorio.usp.br/directbitstream/cbria425a8do-43e5-bird-d718176fdo8I/2954756.pdf $>$. Acesso em 20 abr. 2021.

LIMA, Alana Silva; ALVIM, Haline Gerica de Oliveira. Revisão sobre antiinflamatório não-esteroidais: ácido acetilsalicílico. Revista de Iniciação Científica e Extensão, 2018; I(Esp): I69-74. Disponível em: 〈https://revistasfacesa.senaaires.com.br/index.php/iniciacaocientifica/article/download/69/34/59>. Acesso em 22 abr. 202I.

MIRANDA FILHO, Jorge Paulo de. Cuidados farmacêuticos e os medicamentos isentos de prescrição: uma revisão integrativa da literatura. 2018. 66f. Trabalho de Conclusão de Curso (Graduação) - Universidade Federal de Campina Grande, Cuité, 2018. Disponível em: 
〈http://dspace.sti.ufcg.edu.br:8o8o/jspui/bitstream/riufcg/67or/r/JORGE\%2oPAULO\%2 oDE\%20MIRANDA\%2oFILHO\%20-\%20TCC\%20FARM\%C3\%8ICIA\%202018.pdf>.

Acesso em 04 mar. 202I.

OLIVEIRA, Marialice de. Estudo do perfil de prescrição do Ibuprofeno na Farmácia Escola da Universidade Federal de Ouro Preto. 2019. 65f. Trabalho de Conclusão de Curso (Graduação) - Universidade Federal de Ouro Preto, Ouro Preto, 2019. Disponível em: <https://monografias.ufop.br/bitstream/3540000o/I853/I/MONOGRAFIA_EstudoPerfilP rescri\% $\mathrm{C}_{3 \%} \% \mathrm{~A}_{7 \%} \mathrm{C}_{3 \%} \% \mathrm{~A}_{30 . p d f}>$. Acesso em 26 mar. 2021.

PARGANA, Alexandra Cristina Neves. Reações adversas gastrointestinais Causas, prevenção e tratamento. 2015. 69f. Dissertação (Mestrado) - Universidade do Algarve, Faro, Portugal, 2015. Disponível em:

$\langle$ https://sapientia.ualg.pt/bitstream/ı0400.I/7969/I/Monografia_APargana.pdf $\rangle$. Acesso em 04 maio. 202I.

RANKEL, Sibely Aparecida Oliveira; SATO, Marcelo del Olmo; SANTIAGO, Ronise Martins. Uso irracional dos anti-inflamatórios não esteroidais no Município de Tijucas do Sul, Paraná, Brasil. Revista Visão Acadêmica, Curitiba, v.17, n. 4, out. -dez./2or6. Disponível em: 〈https://revistas.ufpr.br/academica/article/view/50205/31865>. Acesso em 24 mar. 202I.

SANDOVAL, Alline Correia et al. O uso indiscriminado dos anti-inflamatórios não esteroidais (AINES). Revista Científica da Faculdade de Educação e Meio Ambiente.

v. 8, n. 2, p.165 - 176, jul./dez., 2017. Disponível em: <https://docplayer.com.br/827356o6Farmacia-o-uso-indiscriminado-dos-anti-inflamatorios-nao-esteroidais-aines.html>.

Acesso em 22 mar. 2021.

SILVA, Marcos Gontijo da; LOURENÇO, Érica Eugênio. Uso indiscriminado de antiinflamatórios em Goiânia-Go e Bela Vista-Go. Revista Científica do ITPAC, Araguaína, v.7, n. 04, out./2014. Disponível em:

$\langle$ https://assets.unitpac.com.br/arquivos/Revista/74/artigo9.pdf $\rangle$. Acesso em 04 maio 202I.

SOTERIO, Karine Azeredo, SANTOS, Marlise Araújo dos. A automedicação no Brasil e a importância do farmacêutico na orientação do uso racional de medicamentos de venda livre: uma revisão. Revista da Graduação, Porto Alegre, v. 09, n. 02, nov./2016. Disponível em: 〈https://revistaseletronicas.pucrs.br/ojs/index.php/graduacao/article/view/25673>. Acesso em 05 abr. 2021.

SYLVESTER, J. Anti-inflamatórios não-esteroidais. Tradução e supervisão da Comissão de Educação Continuada/Sociedade Brasileira de Anestesiologia. Disponível em: 〈https://www.sbahq.org/resources/pdf/atotw/405.pdf〉. Acesso em 22 abr. 2021

VIEIRA, Fernanda Silva. Automedicação: o uso indiscriminado de anti-inflamatórios e suas implicações para saúde dos idosos. 2017. 4of. Trabalho de Conclusão de Curso 
(Graduação) - Faculdade do Instituto Brasil, Anápolis, 2017. Disponível em: $\langle$ http://fibra.edu.br/wp-content/uploads/2017/o8/TCC-Fernanda-Vieira.pdf $\rangle$. Acesso em 23 mar. 2021. 